\title{
A Better Personalized Image Searching Algorithm
}

\author{
Huang He, Sheng Guang feng, Du Jing jing, Lei Xu, Zhang Tao \\ School of Electronic \& Control Engineering in Chang'an University, Xi'an 710064,China
}

Keywords: image; cluster algorithms; search engines; semantics; information fusion;

\begin{abstract}
Searching engines become the major tool for information retrieval of users with the rapidly increasing Web information. Traditional search engines can' t completely evaluate users' search aims. It will lead to retrieval quality decline and increased cost. The paper proposed a better personalized image searching algorithm. It utilizes relevant feedback and SVM to build user interest model and return the personalized searching results to the users based on the muli-kernel cluster for images. The analysis of experiment results indicate that compared with the traditional searching algorithm the improved algorithm can enhance participation methods of users and solve the gap problem between low-level vision feature and high-level semantics. It can increase the mean recall and precision ratio to $8 \%$ and $10.5 \%$ compared with the traditional searching algorithm based on color. At the same time it can increase them to $13.2 \%$ and $28.56 \%$ respectively compared with the traditional one based on texture.
\end{abstract}

\section{Introduction}

With the rapid developments and applications of information technology as well as image processing technology, society or business can produce vast multimedia information every day. However, how to get useful information from numerous information, especially more and more attention is focused on the retrieval of image information. There are the following problems in the existing search engine[3-4]: (1) The search engine simplifies image search, and it is just taken as a simple and pure text search while ignoring the semantic similarity between the images. (2) When people query a keyword, all the images containing the keywords will be downloaded to the index database as searching result. Then it will return to the user sorted according to the certain algorithms. In this case, without taking into account the user's personal interests, the search engine cannot supply different personalized search results to meet the needs of different users when they use the same key word. The relevant feedback technology is well introduced into the users' intentions in the query system, and it can well describe the users' search aims by clicking and evaluating.

Therefore, in order to solve the above problems, this paper presents a personalized image search based on clustering and user interest model. After system captures the image, the clustering results image will be reclassification to present the image in line with user needs.

\section{Feature Extraction}

Visual characteristics of the image are very important to the contents of image description. The paper selects the local color histogram and global texture features.

2.1 Local Color Feature

In order to achieve a balance between the description of the image object and the control of calculated amount, the local color feature of this paper is to divide the image into nine segment. The visual similarity between local color histograms $\Upsilon, \Psi$ that the two images $\mathrm{x}, \mathrm{y}$ corresponded utilizes kernel function(1) to calculate[5-6]:

$$
K_{1}(x, y)=e^{-\chi^{2}(\Upsilon, \Psi) / \theta}=\prod_{j=1}^{n} \prod_{i=1}^{m} e^{-\chi_{i}^{2}\left(\Upsilon_{j}(i), \Psi_{j}(i)\right) / \theta_{i}}
$$


In which, $\Theta=\left[\theta_{1}, \ldots, \theta_{m}\right]$ represents the average distance of all the images $\chi^{2}$. The formula of $\chi^{2}$ means as follows:

$$
\chi^{2}(u, v)=\frac{1}{2} \sum_{i=1}^{n} \frac{\left(u_{i}-v_{i}\right)^{2}}{u_{i}+v_{i}}
$$

In which $u_{i}$ and $v_{i}$ are the components of the color histograms of the two images. Color histogram kernel function $K_{1}$ can describe the differences between the visual image.

2.2 Global Texture

This paper uses spectrum method to texture feature extraction. 2DGabor wavelet transform is defined by image convolution:

$$
\Gamma_{\mathbf{k}}(\mathbf{z})=\iint I\left(\mathbf{z}^{\prime}\right) \psi_{\mathbf{k}}\left(\mathbf{z}-\mathbf{z}^{\prime}\right) d \mathbf{z}^{\prime}
$$

In which, Gabor filter is defined as:

$$
\psi_{\mathbf{k}}(\mathbf{z})=\frac{\mathbf{k}^{T} \mathbf{k}}{\sigma^{2}} \exp \left(-\frac{\mathbf{k}^{T} \mathbf{k}}{2 \sigma^{2}} \mathbf{z}^{T} \mathbf{z}\right)\left(\exp \left(i \mathbf{k}^{T} \mathbf{z}\right)-\exp \left(-\frac{\sigma^{2}}{2}\right)\right)
$$

In which, $\mathbf{z}=(x, y), \mathbf{k}$ represents the wave vector and satisfies as follows:

$$
\begin{gathered}
\mathbf{k}=\left(k_{v} \cos \varphi_{\mu} \quad k_{v} \sin \varphi_{\mu}\right)^{T} k_{v}=2^{-\frac{v+2}{2} \pi}, \varphi_{\mu}=\mu \frac{\pi}{8} \\
v=0,1,2,3,4 \quad \mu=0, \frac{\pi}{4}, \frac{\pi}{2}, \frac{3 \pi}{4}
\end{gathered}
$$

$v$ and $\mu$ are parameters, and they defined the direction of frequency filter respectively.
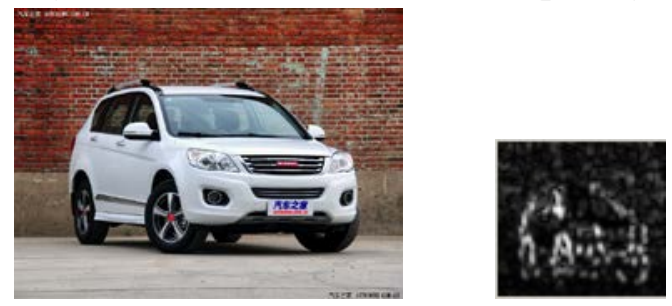

Figure 1 Gabor feature extraction schematic diagram

Using formula (6) calculate the texture similarity of the two images:

$$
K_{2}(x, y)=\prod_{i=1}^{m} e^{-\chi_{i}^{2}\left(h_{i}(x), h_{i}(y)\right) / \sigma_{i}}
$$

In which, $\sigma=\left[\sigma_{1}, \ldots, \sigma_{m}\right]$ represents the average distance of all the images $\chi^{2}$. Formula (2) is used to calculate the average of $\chi^{2}$.

\section{The establishment of multi-core dynamic clustering and user model}

3.1 Multi-core dynamic clustering The main algorithm steps are as follows:

(1) Firstly, to determine the number of clusters category $\mathrm{C}$ and the allowable the max error;

(2) To determine the initialization of the cluster center $W_{i}(k), \quad i=1, \ldots, C$

(3) The input feature vector space is mapped into high-dimensional feature space by using the formula (1) and Equation (6);

(4) By defining some scatter matrix leads to a number of criteria function, which not only reflects the degree of aggregation of the same sample, but also reflects the degree of separation between different classes; Scatter matrix is defined as the first sub-class:

$$
\mathbf{S}_{i}=\sum_{\mathbf{X} \in \chi_{i}}\left(\mathbf{X}-\boldsymbol{\mu}_{i}\right)\left(\mathbf{X}-\boldsymbol{\mu}_{i}\right)^{\mathrm{T}}
$$


In which,

$$
\boldsymbol{\mu}_{i}=\frac{1}{N_{i}} \sum_{y \in \Gamma_{i}} \mathbf{y}
$$

Since the use of trace scatter matrix measure the divergence matrix size is an effective method, it minimizes the scatter matrix trace of the class, and maximizes the trace of the class scatter matrix at the same time. it is in the smallest class scatter matrix trace, but also to maximize the between-class scatter matrix trace. To determine $t r \mathbf{S}_{i}$. and compare the size of several sub-class track, then the largest sub-class track will be divided into two parts. It is divided according to the standard re-cluster similarity criteria. Until it reaches the set number of clusters, cluster output number.

\subsection{The establishment of user interest model}

In this paper, the establishment of user's model is divided into the following steps:

(1) Combining equation (1) and (6) linear. Using equation (8) to calculate the similarity between each image, and the resulting similarity matrix as a multi-core dynamic clustering algorithm inputs;

$$
K(x, y)=\sum_{i=1}^{m} \alpha_{i} K_{i}(x, y), \quad \sum_{i=1}^{m} \alpha_{i}=1
$$

In which, $\alpha_{i} \geq 0, m$ represents the number of kernels.

(2)To complete the cluster of data-basing of sample images by multi-core dynamic clustering algorithm;

(3)Image search is displayed through the search request made by the user. If the displayed image cannot meet the needs of users, users manually select photos they are interested in. After the system capture the image, the image and all the other images which are clustered into one class with it are sent into the SVM network as positive samples. They are trained to determine the classification of the surface to create user interest model. The trained network search sample library images again to sort the images and display the images which users are more interested in, and provide personalized search results. As is shown in Figure 2.

(4) If the results obtained dose not meet the needs of users, return (2).

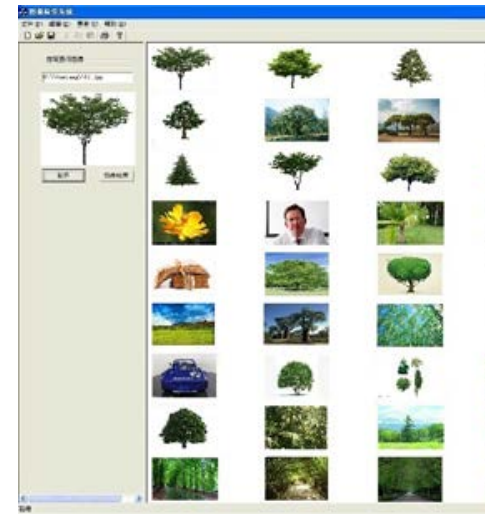

(a) Initial search results

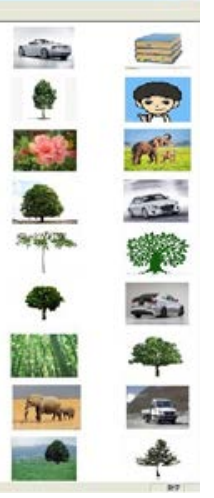

(b) Results SVM training to re-retrieved from the database

Figure 2 Experimental results and analysis

In the experiments, Corel Image library is divided into 20 different categories. Each category contains 100, a total of 2000 images. Multiple sets of keywords are including six groups of tree, elephant, car, flower, face and book. In order to evaluate the effectiveness of the algorithm objectively, using average recall rate and precision rate to evaluate system. Formula is defined as follows:

$$
\begin{gathered}
\text { Average recall rate }(\mathrm{ARR})=\frac{\beta}{\beta+\eta} \\
\text { Precision rate }(\mathrm{PR})=\frac{\beta}{\beta+\omega}
\end{gathered}
$$

In which , $\beta$ represents retrieved picture, $\omega$ represents the picture is not retrieved, $\eta$ represents not retrieved picture.The results are shown in Table 1. 
Table 1 Comparison of traditional content-based retrieval method and the algorithm

\begin{tabular}{cccc}
\hline \multirow{2}{*}{ Keywords } & Only color feature & Only the texture features & Algorithm \\
\cline { 2 - 4 } & ARR/ PR & ARR/ PR & ARR/ PR \\
\hline tree & $93 \% / 91 \%$ & $89 \% / 80 \%$ & $98 \% / 100 \%$ \\
\hline Elephant & $78 \% / 79.2 \%$ & $81 \% / 78.5 \%$ & $85 \% / 84 \%$ \\
\hline Car & $80 \% / 73 \%$ & $64 \% / 51 \%$ & $86 \% / 83 \%$ \\
\hline flower & $66 \% / 58.4 \%$ & $45 \% / 41.3 \%$ & $79 \% / 73.6 \%$ \\
\hline face & $71 \% / 78 \%$ & $56 \% / 48 \%$ & $77 \% / 80 \%$ \\
\hline Book & $72 \% / 60 \%$ & $73 \% / 61 \%$ & $83 \% / 82 \%$
\end{tabular}

As can be seen from the experimental results, since the traditional content-based retrieval method did not establish the user interest model, query results are not satisfactory. While the retrieval method of user model is proposed in this paper, which greatly increases the user's participation mode, that can solve the problem of the gap between underlying visual features and semantic.According to the calculation, in average recall and precision, the proposed algorithm is improved by $8 \%$ and $10.5 \%$ respectively compared with traditional search algorithm based on color and the algorithm is improved by $13.2 \%$ and $28.56 \%$ respectively compared with traditional search algorithm based on texture.

\section{Summary}

For the problem of the poor current search results of search engine, this paper proposed a clustering algorithm based on personalized search and user model. It improved the problem that the traditional SVM needs too many users participate in. It sent the clustering results as positive samples into SVM network to train and adjust the classification face constantly. Then the classification face would be applied to image retrieval of database again. It can be seen that to build user model may be more accurate and comprehensive to find users' interested images through the experiment searching ways.

\section{Acknowledgements}

This work was financially supported by National Natural Science Foundation(61402052, 61203233, 41101357), Natural Science Basic Research Project of Shaanxi Province (2015JM6280) and Fundamental Research Funds for the Central Universities ( 31083216007, 310832151088, 310832151092, 310832151091).

\section{References}

[1] Noruzi, A.. Google Scholar: The New Generation of Citation Indexes[J]. Libri, 2005. 55(4): 170-180.

[2] Gao, Y.,Peng, J., Luo, H, Keim, D., Fan.J. An Interactive Approach for Filtering Out Junk Images from Keyword-Based Goolge Search Results[J]. IEEE Transaction on Circuits and Systems for Video Technology, 2009,19(12): 1851-1865.

[3] Kun Yan, Xiaoyi Feng, He Huang. Shaochong Fan. A novel algorithm for filtering out junk images interactively from web search results[C]. IEEE International Conference on Computer Science and Information Technology, 2010, 8:195 - 199.

[4] L.Zhang, F.Lin. Support Vector Machine Learning for Image Retrieval[C]. IEEE Conference on Image Processing, 2001, 2:721-724

[5] J. Fan, Y.Gao, H. Luo, Integrating concept ontology and multi-task learning to ahieve more effective classifier training for multi-level image annotation[J]. IEEE Transaction on Image 
Processing, 2008, 17 (3) : 407 - 426

[6] Zhi-yong Teng Sheng-hua Du Kun Zou Mou-yan. Colorization Based on Unevenness in Chrominance Blending[C]. IEEE Conference on Intelligent Computer Communication and Processing, 2008, 17 (3): :407 - 426 